\title{
Existencia y unicidad para un sistema acoplado de ecuaciones de onda con término disipativo débil
}

\author{
Andrés Guardia Cayo ${ }^{1}$ y Alfonso Pérez Salvatierra ${ }^{2}$
}

Resumen: En este trabajo se estudia la existencia, unicidad y regularidad de la solución de un sistema acoplado de ecuaciones de onda vía el método de la teoría de semigrupos de operadores lineales.

Palabras clave: sistema acoplado; ecuación de onda; generador infinitesimal.

\section{Existence and uniqueness for a coupled system of wave equations with weak dissipative term}

\begin{abstract}
In this work we study the existence, uniqueness and regularity of the solution of a coupled system of wave equations by the method of the theory of linear operators semigroups.
\end{abstract}

Keywords: coupled system; wave equation; infinitesimal generator.

Recibido: 05/05/2019. Aceptado: 20/11/2019. Publicado online: 26/12/2019.

(CLos autores. Este artículo es publicado por la Revista PESQUIMAT de la Facultad de Ciencias Matemáticas, Universidad Nacional Mayor de San Marcos. Este es un artículo de acceso abierto, distribuido bajo los términos de la licencia Creative Commons Atribucion-No Comercia-Compartir Igual 4.0 Internacional.(http://creativecommons.org/licenses/by-nc-sa/4.0/) que permite el uso no comercial, distribución y reproducción en cualquier medio, siempre que la obra original sea debidamente citada. Para información, por favor póngase en contacto con revistapesquimat.matematica@unmsm.edu.pe

\footnotetext{
${ }^{1}$ UNMSM, Facultad de Ciencias Matemáticas, e-mail:aguardiac@unmsm.edu.pe

${ }^{2}$ UNMSM, Facultad de Ciencias Matemáticas, e-mail: aperezs@unmsm.edu.pe
} 


\section{Introducción}

En el presente artículo vamos a establecer la existencia y unicidad de solución del sistema acoplado de ecuaciones de onda formulado por el siguiente modelo:

$$
\begin{aligned}
u_{t t}-\Delta u+u_{t}+\alpha(u-v) & =0 \text { en } \Omega \times] 0, \infty[ \\
v_{t t}-\Delta v-\alpha(u-v) & =0 \text { en } \Omega \times] 0, \infty[
\end{aligned}
$$

con condiciones de frontera

$$
u=v=0 \text { sobre } \Gamma \times] 0, \infty[
$$

y condiciones iniciales

$$
\begin{gathered}
u(x, 0)=u_{0}(x), u_{t}(x, 0)=u_{1}(x) \text { en } \Omega \\
v(x, 0)=v_{0}(x), v_{t}(x, 0)=v_{1}(x) \text { en } \Omega
\end{gathered}
$$

donde $\Omega$ es un conjunto abierto, acotado de $\mathbb{R}^{n} ; n \geq 1$ con frontera $\Gamma=\partial \Omega$ bien regular y $\alpha$ es una constante real positiva

El modelo dado en (1)-(5), puede usarse para describir la evolución de un sistema que consta de dos membranas elásticas sujetas a una fuerza elástica que atrae una membrana a la otra con un coeficiente $\alpha>0$. El término $u_{t}$, actúa en la primera membrana como estabilizador, mas detalles al respecto, ver [3].

Los términos disipativos internas, ayudan bastante para que la energía de la ecuación tenga un comportamiento asintótico, como también los efectos térmicos o la memoria actúan como términos disipativos, es de observar, cuando se tiene un sistema de dos ecuaciones de tipo ondas, se requerirá de dos términos disipativos uno en cada ecuación para asegurar un decaimiento exponencial; pero si solo tiene un solo término disipativo interno en una de las ecuaciones, es posible que tengan solo un decaimiento polinomial. En [4], F. Alabau et al., trabajaron un sistema acoplado de dos ecuaciones de ondas con un solo término disipativo en forma general dado por,

$$
\mid \begin{gathered}
\left.u_{t t}-A_{1} u+B u_{t}+\alpha v=0 \text { en } \Omega \times\right] 0, \infty[ \\
\left.v_{t t}-A_{2} v+\alpha v=0 \text { en } \Omega \times\right] 0, \infty[ \\
u=v=0 \text { sobre } \Gamma \times] 0, \infty[ \\
u(x, 0)=u_{0}(x), u_{t}(x, 0)=u_{1}(x), x \in \Omega \\
v(x, 0)=v_{0}(x), v_{t}(x, 0)=v_{1}(x), x \in \Omega
\end{gathered}
$$

donde $A_{1}, A_{2}$ y $B$ son operadores lineales auto-adjuntos positivos, $B$ operador acotado.

Los autores muestran que el sistema acoplado (6) es disipativo; pero el semigrupo correspondiente no es exponencialmente estable. Además, prueban que la solución del sistema anterior decae polinomialmente a cero a medida que el tiempo tiende al infinito. 


\section{Preliminares}

Enunciamos resultados esenciales de la teoría de semigrupos lineales, que serán útiles en el desarrollo de este trabajo. Omitimos las demostraciones, sin embargo el lector interesado puede hallar las demostraciones respectivas en [5] y [6]. Con referencia a los espacios de Sobolev ver [1] y [2].

Definición 1 Sea $X$ un espacio de Hilbert y $T: D(T) \subset X \rightarrow X$ un operador lineal; no acotado en $X$, el conjunto resolvente $\rho(T)$ se define como

$$
\rho(T):=\left\{\lambda \in \mathbb{C}:(\lambda I-T) \text { es invertible } y(\lambda I-T)^{-1} \text { es un operador acotado en } X\right\}
$$

El operador lineal acotado

$$
R(\lambda ; T):=(\lambda I-T)^{-1}
$$

con $\lambda \in \rho(T)$, se llama resolvente de $T$.

Teorema 1 (Hille-Yosida) Sea $X$ un espacio de Hilbert y $\mathcal{A}$ un operador lineal no limitado es generador infinitesimal de un semigrupo $C_{0}$ de contracciones, si y solo si

i) $\mathcal{A}$ es un operador cerrado y $\overline{D(\mathcal{A})}=X$

ii) $\mathbb{R}^{+} \subset \rho(\mathcal{A})$ y $\left\|(\lambda I-\mathcal{A})^{-1}\right\| \leq \frac{1}{\lambda}, \forall \lambda>0$.

Demostración. Ver [6].

Teorema 2 (Lummer-Phillips) Sea $\mathcal{A}$ un operador lineal con dominio denso en un espacio de Hilbert $X$.

i) Si $\mathcal{A}$ es disipativo y existe $\lambda$ tal que $\operatorname{Im}(\lambda I-\mathcal{A})=X$, entonces $\mathcal{A}$ es un generador infinitesimal de un semigrupo de clase $C_{0}$ de contracciones.

ii) Si $A$ un generador infinitesimal de un semigrupo de clase $C_{0}$ de contracciones sobre el espacio $X$, entonces $\operatorname{Im}(\lambda I-\mathcal{A})=X$ para todo $\lambda>0$, y $\mathcal{A}$ es un operador disipativo.

Demostración. Ver [5].

Teorema 3 Sea $X$ un espacio de Banach y $\mathcal{A}$ un operador lineal (no acotado), disipativo y con dominio denso en $X$. Si $0 \in \rho(A)$, entonces $\mathcal{A}$ es el generador infinitesimal de un semigrupo $C_{0}$ de contracciones $S(t)=e^{\mathcal{A t}}$ sobre $X$.

Demostración. Ver [6].

Definición 2 Sea $X$ un espacio de Banach, $\mathcal{A}$ un operador lineal no acotado, $D(\mathcal{A}) \subset X$ y $U_{0} \in X$ un dato inicial. El Problema de Valor Inicial

$$
\mid \begin{aligned}
\frac{d U}{d t} & =\mathcal{A} U, \quad t>0 \\
U(0) & =U_{0}
\end{aligned}
$$

se denomina Problema Abstracto de Cauchy. 
Teorema 4 Sea $\mathcal{H}$ un espacio de Hilbert y el operador lineal $\mathcal{A}: D(\mathcal{A}) \subset \mathcal{H} \rightarrow \mathcal{H}$ el generador infinitesimal de un semigrupo de clase $C_{0}$.

i) Si $U_{0} \in D(\mathcal{A})$, entonces el problema (7) tiene una única solución $U(t)=S(t) U_{0}$ satisfaciendo

$$
U \in C^{1}([0, \infty) ; \mathcal{H}) \cap C([0, \infty) ; D(\mathcal{A}))
$$

ii) Si $U_{0} \in \mathcal{H}$, entonces el problema (7) tiene una única solución $U(t)=S(t) U_{0}$ satisfaciendo

$$
U \in C([0, \infty) ; \mathcal{H}) \cap C^{1}((0, \infty) ; \mathcal{H}) \cap C((0, \infty) ; D(\mathcal{A}))
$$

Demostración. Ver [5].

Teorema 5 Sea $X$ un espacio de Hilbert y $\mathcal{A}: D(\mathcal{A}) \subset X \longrightarrow X$ un operador lineal disipativo con $\operatorname{Im}(I-\mathcal{A})=X$. Si $X$ es reflexivo, entonces $\overline{D(\mathcal{A})}=X$.

Demostración. Ver [5].

\section{Existencia y unicidad}

Efectuando el cambio de variable $\varphi=u_{t}, \psi=v_{t} \mathrm{y}$ denotando con $U=\left(u, u_{t}, v, v_{t}\right) \mathrm{y}$ $U_{0}=\left(u_{0}, u_{1}, v_{0}, v_{1}\right)$ el sistema (1)-(5) se puede reescribir como el problema de Cauchy

$$
\mid \begin{aligned}
& \frac{d U}{d t}=\mathcal{A} U, \text { para } t>0 \\
& U(0)=U_{0} .
\end{aligned}
$$

donde $\mathcal{A}: D(\mathcal{A}) \subset \mathcal{H} \longrightarrow \mathcal{H}$ es el operador diferencial definido por

$$
\mathcal{A}=\left(\begin{array}{cccc}
0 & I & 0 & 0 \\
\Delta-\alpha I & -I & \alpha I & 0 \\
0 & 0 & 0 & I \\
\alpha I & 0 & \Delta-\alpha I & 0
\end{array}\right)
$$

siendo $0, I$, y $\Delta$ los operadores nulo, identidad y Laplaciano respectivamente.

Con el fin de efectuar la formulación del semigrupo asociado al sistema (1)-(5), definimos la energía del sistema

$$
E(t):=\frac{1}{2} \int_{\Omega}\left|u_{t}\right|^{2} d x+\frac{1}{2} \int_{\Omega}\left|v_{t}\right|^{2} d x+\frac{1}{2} \int_{\Omega}|\nabla u|^{2} d x+\frac{1}{2} \int_{\Omega}|\nabla v|^{2} d x+\frac{\alpha}{2} \int_{\Omega}|u-v|^{2} d x
$$

a fin de poder determinar el espacio de Hilbert $\mathcal{H}$.

Proposición 1 Si $E(t)$ es la energía asociada al sistema (1)-(5), entonces

$$
\frac{d}{d t} E(t)=-\int_{\Omega}\left|u_{t}\right|^{2} d x
$$

Demostración. Multiplicando formalmente la ecuación (1) y (2) por $u_{t}, v_{t} \in L^{2}(\Omega)$ respectivamente e integrando sobre $\Omega$, obtenemos

$$
\int_{\Omega} u_{t t} u_{t} d x-\int_{\Omega} \Delta u u_{t} d x+\int_{\Omega} u_{t} u_{t} d x+\alpha \int_{\Omega}(u-v) u_{t} d x=0
$$




$$
\int_{\Omega} v_{t t} v_{t} d x-\int_{\Omega} v_{t} \Delta v d x-\alpha \int_{\Omega}(u-v) v_{t} d x=0
$$

Aplicando el Teorema de Green, y teniendo en cuenta que $u, v$ se anula en $\Gamma=\partial \Omega$, obtenemos

$$
\frac{1}{2} \frac{d}{d t} \int_{\Omega}\left|u_{t}\right|^{2} d x+\int_{\Omega} \nabla u \nabla u_{t} d x+\int_{\Omega}\left|u_{t}\right|^{2} d x-\alpha \int_{\Omega}(u-v) u_{t} d x=0
$$

$\mathrm{y}$

$$
\frac{1}{2} \frac{d}{d t} \int_{\Omega}\left|v_{t}\right|^{2} d x+\int_{\Omega} \nabla v \nabla v_{t} d x-\alpha \int_{\Omega}(u-v) v_{t} d x=0 .
$$

Sumando (14), (15) y agrupando convenientemente se obtiene

$$
\begin{aligned}
\frac{1}{2} \frac{d}{d t} \int_{\Omega}\left|u_{t}\right|^{2} d x+\frac{1}{2} \frac{d}{d t} \int_{\Omega}\left|v_{t}\right|^{2} d x+\frac{1}{2} & \frac{d}{d t} \int_{\Omega}|\nabla u|^{2} d x+\frac{1}{2} \frac{d}{d t} \int_{\Omega}|\nabla v|^{2} d x \\
& +\frac{\alpha}{2} \frac{d}{d t} \int_{\Omega}|u-v|^{2} d x=-\int_{\Omega}\left|u_{t}\right|^{2} d x .
\end{aligned}
$$

teniendo en cuenta que

$$
E(t):=\frac{1}{2} \int_{\Omega}\left|u_{t}\right|^{2} d x+\frac{1}{2} \int_{\Omega}\left|v_{t}\right|^{2} d x+\frac{1}{2} \int_{\Omega}|\nabla u|^{2} d x+\frac{1}{2} \int_{\Omega}|\nabla v|^{2} d x+\frac{\alpha}{2} \int_{\Omega}|u-v|^{2} d x
$$

de (16) y (17) se concluye

$$
\frac{d}{d t} E(t)=-\int_{\Omega}\left|u_{t}\right|^{2} d x \leq 0, \forall t \geq 0 .
$$

Por tanto, la energía del sistema es decreciente.

Observación 1 Del resultado de la proposición 1, para que la energía esté bien definida, se establece que la solución $U=(u, \varphi, v, \psi)$ debe satisfacer

$$
U \in H_{0}^{1}(\Omega) \times L^{2}(\Omega) \times H_{0}^{1}(\Omega) \times L^{2}(\Omega)
$$

De este modo se define el espacio de fase asociado al sistema (8) dado por

$$
\mathcal{H}=H_{0}^{1}(\Omega) \times L^{2}(\Omega) \times H_{0}^{1}(\Omega) \times L^{2}(\Omega) .
$$

El espacio $\mathcal{H}$, provisto del siguiente producto interno

$$
\langle U, \widetilde{U}\rangle_{\mathcal{H}}=\int_{\Omega} \nabla u \cdot \nabla \widetilde{u} d x+\int_{\Omega} \varphi \widetilde{\varphi} d x+\int_{\Omega} \nabla v \cdot \nabla \widetilde{v} d x+\int_{\Omega} \psi \widetilde{\psi} d x+\alpha \int_{\Omega}(u-v)(\widetilde{u}-\widetilde{v}) d x
$$

Con $U=(u, \varphi, v, \psi), \widetilde{U}=(\widetilde{u}, \widetilde{\varphi}, \widetilde{v}, \widetilde{\psi}) \in \mathcal{H}$; es un espacio de Hilbert, con la norma dada por

$$
\|U\|_{\mathcal{H}}^{2}=\|\nabla u\|_{L^{2}(\Omega)}^{2}+\|\varphi\|_{L^{2}(\Omega)}^{2}+\|\nabla v\|_{L^{2}(\Omega)}^{2}+\|\psi\|_{L^{2}(\Omega)}^{2}+\alpha\|u-v\|_{L^{2}(\Omega)}^{2} .
$$

El dominio del operador $\mathcal{A}$ es el conjunto sobre el cual el operador está bien definido sobre el espacio de fase, esto es,

$$
D(\mathcal{A})=\{U \in \mathcal{H} \mid \mathcal{A} U \in \mathcal{H}\}
$$

teniendo en cuenta el operador $\mathcal{A}$ definido por (9) y el vector $U=(u, \varphi, v, \psi)$ se deduce

$$
D(\mathcal{A})=\left[\left(H^{2}(\Omega) \cap H_{0}^{1}(\Omega)\right) \times H_{0}^{1}(\Omega)\right]^{2} .
$$

De las inmersiones de $H_{0}^{1}(\Omega) \cap H^{2}(\Omega) \stackrel{C}{\hookrightarrow} H_{0}^{1}(\Omega)$ y $H_{0}^{1}(\Omega) \stackrel{C}{\hookrightarrow} L^{2}(\Omega)$ se tiene que $\overline{D(\mathcal{A})}=\mathcal{H}$. 
De este modo con los resultados anteriores, el sistema (1)-(5) es equivalente a mostrar el problema de Cauchy dado por (8) sobre el espacio $\mathcal{H}$, con $U=\left(u, u_{t}, v, v_{t}\right)^{T}$ y $U_{0}=\left(u_{0}, u_{1}, v_{0}, v_{1}\right)^{T} \in$ $D(\mathcal{A})$ donde " $T$ " denota la transpuesta de $U$ y $U_{0}$ respectivamente siendo $\mathcal{A}$ el operador diferencial definido en $(9)$.

En lo que sigue se probará, usando el Teorema 3, que $\mathcal{A}$ es el generador infinitesimal de un semigrupo de contracciones $\{S(t)\}_{t \geq 0}$ de clase $C_{0}$ sobre el espacio de Hilbert $\mathcal{H}$. Con este fin, desarrollaremos los siguientes resultados con respecto del operador diferencial $\mathcal{A}$.

Proposición 2 El operador $\mathcal{A}: D(\mathcal{A}) \subset \mathcal{H} \rightarrow \mathcal{H}$ es disipativo.

Demostración. En efecto, sea $U=(u, \varphi, v, \psi)^{T} \in D(\mathcal{A})$, y el operador $\mathcal{A}$ definido por (9), entonces

$$
\mathcal{A} U=(\varphi, \Delta u-\varphi-\alpha u+\alpha v, \psi, \Delta v+\alpha u-\alpha v)^{T} \in \mathcal{H} .
$$

Luego

$$
\langle\mathcal{A} U, U\rangle_{\mathcal{H}}=\int_{\Omega} \nabla \varphi \nabla u d x+\int_{\Omega} \varphi \Delta u d x-\int_{\Omega} \varphi \varphi d x+\int_{\Omega} \nabla \psi \nabla v d x+\int_{\Omega} \psi \Delta v d x .
$$

Aplicando el teorema de Green, se obtiene

$$
\langle\mathcal{A} U, U\rangle_{\mathcal{H}}=-\int_{\Omega}|\varphi|^{2} d x
$$

de donde

$$
\operatorname{Re}\langle\mathcal{A} U, U\rangle_{\mathcal{H}}=-\int_{\Omega}|\varphi|^{2} d x \leq 0
$$

Por tanto, el operador $\mathcal{A}$ es disipativo.

Proposición $30 \in \rho(\mathcal{A})$

Demostración. Dado $F=(f, g, h, q) \in \mathcal{H}=H_{0}^{1}(\Omega) \times L^{2}(\Omega) \times H_{0}^{1}(\Omega) \times L^{2}(\Omega)$, por demostrar que existe un único $U=(u, \varphi, v, \psi)^{T} \in D(\mathcal{A})$ tal que

$$
\mathcal{A} U=-F
$$

De (25), en términos de sus componentes se tiene

$$
\begin{aligned}
\varphi & =-f \in H_{0}^{1}(\Omega) \\
\Delta u-\varphi-\alpha u+\alpha v & =-g \in L^{2}(\Omega) \\
\psi & =-h \in H_{0}^{1}(\Omega) \\
\Delta v+\alpha u-\alpha v & =-q \in L^{2}(\Omega) .
\end{aligned}
$$

reduciendo variables en (26)-(29) se obtiene el siguiente sistema equivalente

$$
\begin{aligned}
& \Delta u-\alpha u+\alpha v=-f-g \in L^{2}(\Omega) \\
& \Delta v+\alpha u-\alpha v=-q \in L^{2}(\Omega)
\end{aligned}
$$

Pasando a su formulación débil, esto es, multiplicando (30) y (31) por $z, w \in H_{0}^{1}(\Omega)$ respectivamente, integrando sobre $\Omega$ y aplicando Green, se obtiene

$$
\begin{aligned}
\int_{\Omega} \nabla z \cdot \nabla u d x+\int_{\Omega} \alpha u z d x-\int_{\Omega} \alpha v z d x & =\int_{\Omega} f z d x+\int_{\Omega} g z d x \\
\int_{\Omega} \nabla w \cdot \nabla v d x-\int_{\Omega} \alpha u w d x+\int_{\Omega} \alpha v w d x & =\int_{\Omega} q w d x
\end{aligned}
$$


Sumando (32) y (33), obtenemos la siguiente ecuación

$$
\int_{\Omega} \nabla z \cdot \nabla u d x+\int_{\Omega} \nabla w \cdot \nabla v d x+\alpha \int_{\Omega}(u-v)(z-w) d x=\int_{\Omega}((f+g) z+q w) d x .
$$

A fin de garantizar la solución del sistema (26)-(29), para $\vartheta=(u, v)$ y $\xi=(z, w)$ con $\vartheta, \xi \in H_{0}^{1}(\Omega) \times H_{0}^{1}(\Omega)$ definimos la forma bilineal

$$
\mathcal{B}:\left[H_{0}^{1}(\Omega) \times H_{0}^{1}(\Omega)\right]^{2} \longrightarrow \mathbb{R}
$$

donde

$$
\mathcal{B}(\vartheta, \xi)=\int_{\Omega} \nabla z \cdot \nabla u d x+\int_{\Omega} \nabla w \cdot \nabla v d x+\alpha \int_{\Omega}(u-v)(z-w) d x,
$$

y la forma lineal

$$
\mathcal{L}: H_{0}^{1}(\Omega) \times H_{0}^{1}(\Omega) \rightarrow \mathbb{R}
$$

definida por

$$
\mathcal{L}(z, w):=\int_{\Omega}((f+g) z+q w) d x=\int_{\Omega}(f+g) z d x+\int_{\Omega} q w d x .
$$

Afirmación 1 En el espacio $W=H_{0}^{1}(\Omega) \times H_{0}^{1}(\Omega)$ con la norma dada por

$$
\|(u, w)\|_{W}^{2}=\int_{\Omega}|\nabla u|^{2} d x+\int_{\Omega}|\nabla v|^{2} d x+\alpha \int_{\Omega}|u-v|^{2} d x ; \quad \vartheta=(u, v) \in W
$$

la forma bilineal $\mathcal{B}(\vartheta, \xi)$ definido en (35) es continua y coerciva.

En efecto,

i) $\mathcal{B}(\vartheta, \xi)$ es continua

Sea $\vartheta=(u, v), \xi=(z, w) \in H_{0}^{1}(\Omega) \times H_{0}^{1}(\Omega)$, aplicando la desigualdad triangular y Cauchy-Schwarz se obtiene

$$
\begin{aligned}
|\mathcal{B}(\vartheta, \xi)| \leq & \int_{\Omega}|\nabla z \cdot \nabla u| d x+\int_{\Omega}|\nabla w \cdot \nabla v| d x+\alpha\left(\int_{\Omega}|u-v||z-w| d x\right) \\
\leq & {\left[\left(\int_{\Omega}|\nabla u|^{2} d x\right)\left(\int_{\Omega}|\nabla z|^{2} d x\right)\right]^{1 / 2} } \\
+ & {\left[\left(\int_{\Omega}|\nabla v|^{2} d x\right)\left(\int_{\Omega}|\nabla w|^{2} d x\right)\right]^{1 / 2} } \\
+ & {\left[\alpha^{2}\left(\int_{\Omega}|u-v|^{2} d x\right)\left(\int_{\Omega}|z-w|^{2} d x\right)\right]^{1 / 2} }
\end{aligned}
$$

teniendo en cuenta que

$$
\sqrt{\lambda_{1}}+\sqrt{\lambda_{2}}+\cdots+\sqrt{\lambda_{n}} \leq n \sqrt{\lambda_{1}+\lambda_{2}+\cdots+\lambda_{n}}, \text { para todo } n \in \mathbb{N}
$$

obtenemos

$$
\begin{aligned}
|\mathcal{B}(\vartheta, \xi)| \leq 3\left(\int_{\Omega}|\nabla u|^{2} d x+\int_{\Omega}|\nabla v|^{2} d x+\alpha \int_{\Omega}|u-v|^{2} d x\right)^{1 / 2} & \\
& \times\left(\int_{\Omega}|\nabla z|^{2} d x+\int_{\Omega}|\nabla w|^{2} d x+\alpha \int_{\Omega}|z-w|^{2} d x\right)^{1 / 2} .
\end{aligned}
$$

Por tanto

$$
|\mathcal{B}(\vartheta, \xi)| \leq 3\|\vartheta\|_{W}\|\xi\|_{W}
$$


ii) $\mathcal{B}(\vartheta, \xi)$ es coerciva

En efecto,

$$
\begin{aligned}
\mathcal{B}(\vartheta, \vartheta) & =\mathcal{B}((u, v),(u, v)) \\
& =\int_{\Omega} \nabla u \cdot \nabla u d x+\int_{\Omega} \nabla v \cdot \nabla v d x+\alpha \int_{\Omega}(u-v)(u-v) d x \\
& =\int_{\Omega}|\nabla u|^{2} d x+\int_{\Omega}|\nabla v|^{2} d x+\alpha \int_{\Omega}|u-v|^{2} d x=\|\vartheta\|_{W}^{2} .
\end{aligned}
$$

de donde

$$
\mathcal{B}(\vartheta, \vartheta) \geq 1 \cdot\|\vartheta\|_{W}^{2}
$$

Con lo que queda establecida la afirmación.

De modo similar para $z, w \in H_{0}^{1}(\Omega)$ el funcional

$$
\mathcal{L}: H_{0}^{1}(\Omega) \times H_{0}^{1}(\Omega) \rightarrow \mathbb{R}
$$

definida en (36) es lineal y continua.

Luego, por el Lema de Lax-Milgram, existe una única solución

$$
\xi=(z, w) \in H_{0}^{1}(\Omega) \times H_{0}^{1}(\Omega)
$$

para el problema variacional

$$
\mathcal{B}(\vartheta, \xi)=\mathcal{L}(\xi)
$$

Esto es

$$
\mathcal{B}((u, v),(z, w))=\int_{\Omega} \nabla z \cdot \nabla u d x+\int_{\Omega} \nabla w \cdot \nabla v d x+\alpha \int_{\Omega}(u-v)(z-w) d x,
$$

es decir, $\vartheta=(u, v)$ satisface el sistema (26) y (29).

De este modo se concluye que existe un único

$$
U=(u, \varphi, v, \psi)^{T} \in D(\mathcal{A}),
$$

tal que $\mathcal{A} U=F$, así el operador $\mathcal{A}^{-1}$ existe. Y mas aún existe una constante $C>0$ que depende solamente del dominio de $\mathcal{A}$, tal que

$$
\left\|\mathcal{A}^{-1} F\right\|_{\mathcal{H}} \leq C\|F\|_{\mathcal{H}}, \forall F \in \mathcal{H}
$$

de donde se sigue que

$$
\left\|\mathcal{A}^{-1}\right\|_{\mathcal{H}} \leq C
$$

luego $\mathcal{A}^{-1}$ es acotado, por tanto $0 \in \rho(\mathcal{A})$.

Teorema 6 El operador $\mathcal{A}$ definido en (9) es un generador infinitesimal de un semigrupo de clase $C_{0}$ de contracciones sobre $\mathcal{H}$.

Demostración. En efecto, tenemos que $D(\mathcal{A})$ es denso en $\mathcal{H}$. De las Proposiciones 2 y 3 el operador $\mathcal{A}$ es disipativo, $0 \in \rho(\mathcal{A})$. Luego teniendo en cuenta el Teorema 3 , se concluye que el operador $\mathcal{A}$ es un generador infinitesimal de un semigrupo de clase $C_{0}$ de contracciones sobre el espacio $\mathcal{H}$. En el siguiente teorema establecemos el resultado sobre existencia y unicidad de soluciones. 
Teorema 7 Si $U_{0} \in D(\mathcal{A})$, es decir, $u_{0}, v_{0} \in H^{2}(\Omega) \cap H_{0}^{1}(\Omega)$ y $u_{1}, v_{1} \in H_{0}^{1}(\Omega)$, entonces el problema (8) posee una única solución $U(t)=S(t) U_{0}$ tal que

$$
U \in C\left(\mathbb{R}^{+} ; D(\mathcal{A})\right) \cap C^{1}\left(\mathbb{R}^{+} ; \mathcal{H}\right)
$$

esto es,

$$
u(t), v(t) \in H^{2}(\Omega) \cap H_{0}^{1}(\Omega), \quad u_{t}(t), v_{t}(t) \in H_{0}^{1}(\Omega) \text { y } u_{t t}(t), v_{t t}(t) \in L^{2}(\Omega) .
$$

Demostración. La demostración, se sigue a partir del Teorema 4.

\section{Conclusión}

En este artículo estudiamos un sistema acoplado de ecuaciones de ondas que consiste en dos membranas elásticas sometidas a una fuerza elástica, que se unen entre sí a través del coeficiente $\alpha$. Utilizamos la teoría de semigrupos lineales para demostrar que el sistema (1)-(5) es bien puesto.

El método usado para obtener la existencia y unicidad del sistema acoplado de ecuaciones de onda con término disipativo débil, es uno de los muchos métodos que existen, sin embargo este método es mas conveniente por su fácil manejo y además nos ayuda con los cálculos adicionales con el fin de estudiar la estabilidad del semigrupo asociado al sistema.

Por otro lado el estudio del sistema planteado nos indica que con sólo un término disipativo en una de las ecuaciones es suficiente para obtener una solución fuerte al sistema planteado.

\section{Referencias bibliográficas}

[1] Adams, R. A. (1975). Sobolev Spaces. New York, United States of America: Academic Press.

[2] Brezis, H. (1983). Analyse Fonctionnelle - Théorie et Applications. Francia, Paris: Mason.

[3] Love, A. E. H. (1942). Mathematical Theory of Elasticity. New York, United States of America: Dover Publications, .

[4] Fatiha Alabau, P. (2002). Indirect internal stabilization of weakly coupled evolution equations. Journal of Evolution Equations, 2(29), 127 - 150 .

[5] Pazy, A. (1983). Semigroups of Linear Operators and Applications to Partial Differential Equations. New York, United States of America: Springer-Verlag.

[6] Rivera, J. E. M. (2007). Estabilização de Semigrupos e Aplicações. Petrópolis, Brasil: Laboratório Nacional de Computação Científica. 